\title{
Numerical simulation of tribosystems of the high-force diesel engine taking into account the rheological characteristics of a lubricant
}

\author{
K. Gavrilov, E. Zadorozhnaya \& A. Doikin \\ South Ural State University, Russia
}

\begin{abstract}
To create a modern high performance engine requires considering many design and operational factors. The working conditions of the engine tribosystems, in particular, of the piston-cylinder tribosystem are characterized by time and direction of the load (non-stationary loaded tribosystems). Moreover, they are influenced by the usage of new materials and lubricants with improved tribological properties. Non-stationarity of the piston-cylinder tribosystem combined with severe temperature conditions and a variety of factors make it difficult to create a numerical model and calculate the dynamics of the tribosystem. The paper presents a method of calculation of hydromechanical characteristics of the piston-cylinder tribosystem based on the solution of the Reynolds equation, which takes into account the rheological characteristics of the lubricant (non-Newtonian properties). We describe the rheological model of the lubricant that takes into account the dependence of viscosity from temperature, hydrodynamic pressure and shear rate. The task of the piston dynamics on the lubricating layer in the cylinder of the diesel engine $\mathrm{ChN}$ 13/15 was solved. The influence of non-Newtonian properties on design characteristics of tribosystem was shown. The achieved results can be applied for the design of a diesel engine.

Keywords: piston skirt-cylinder liner tribosystem, non-Newtonian properties, hydromechanical characteristics.
\end{abstract}

\section{Introduction}

The reliability of many machines and mechanisms is mainly determined by the reliability of friction pairs (tribosystems). In this regard, the development of 
the improved simulation methods remains a significant problem. The following methods to solve the dynamics task and the hydrodynamic lubrication task of sliding bearings are used: by determining the hydrodynamic pressure field in a thin lubricating layer between friction surfaces; by analyzing the dynamics of their relative motion; by evaluating the temperature parameters of the lubricating layer for a period of loading.

However, these methods need improvement as to describe real physical processes it is necessary to create complex computational models with the maximum number of factors.

Recently, researchers have paid special attention to the rheological properties of lubricants [1-5]. Therefore, the construction of the rheological models of modern oils reflecting the dependence of viscosity on temperature and pressure in a thin lubricating layer and on the shear rate is of great importance for the simulation of heavy-loaded bearings.

Solving these problems is essential if we want to improve the reliability of friction units that satisfy modern requirements.

\section{Rheological model of a lubricant}

At present, the improvement of bearings design of internal combustion engines and the technology of motor oil production cause most loaded sliding bearings to operate at minimum film thickness [5-7], which is about $1 \mu \mathrm{m}$ in a steady state and is less for low rotation frequency of the journal. This thickness is comparable to twice the height of surface roughness of friction. This time the life cycle of the same unit of friction can vary from 3 to 5 times using different motor oils.

With the support of experimental and theoretical research, it can be argued that the variation of friction conditions leads to a change in the mechanisms of friction and wear. In this case, the change of rheological properties depending on the lubricant film thickness, the contact pressure, the surface roughness, the highly viscous boundary layer and the individual properties of the lubricant plays a major role.

Recently, the works taking into account the presence of a highly viscous boundary layer adsorbed on the surfaces of friction have appeared $[8,9]$.

Thus, the need for a comprehensive rheological model lubricant arose. This model must take into account both the viscosity dependent on the temperature lubrication, hydrodynamic pressure in a thin lubricating layer, shear, changing dramatically the thickness of the layer, relaxation (delay of viscosity change with rapid growth of hydrodynamic pressures) and highly viscous boundary layer. The following viscosity model [8] was proposed:

$$
\mu^{*}(T, p, \dot{\gamma})=\left\{\begin{array}{l}
\mu_{1} \cdot C_{1} e^{\left(C_{2} /\left(T_{p}+C_{3}\right)\right)+\beta\left(T_{p}\right) \cdot p}, 1 \leq \dot{\gamma} \leq 10^{2} ; \\
\left(I_{2}\right)^{\left(n\left(T_{p}\right)-1\right) / 2} \cdot C_{1} e^{\left(C_{2} / T_{p}+C_{3}\right)+\beta\left(T_{p}\right) \cdot p}, 10^{2} \leq \dot{\gamma} \leq 10^{6} ; \\
\mu_{2} \cdot C_{1} e^{\left(C_{2} / T_{p}+C_{3}\right)+\beta\left(T_{p}\right) \cdot p}, \dot{\gamma}>10^{6},
\end{array}\right.
$$


where $\dot{\gamma}=\sqrt{I_{2}}, \quad I_{2}=\left(\partial V_{x} / \partial y\right)^{2}+\left(\partial V_{z} / \partial y\right)^{2}-$ the second invariant of shear rates; $n$-parameter that characterizes the degree of non-Newtonian behavior of lubricants; $\beta(T)$ - piezo-coefficient of viscosity of lubricant; $p$-hydrodynamic pressure; $T$ - the temperature of the lubricating layer; $C_{1}, C_{2}, C_{3}$ - constants.

At section 1 in the range of shear rates from 1 to $10^{2} \mathrm{~s}^{-1}$ oil behaves as a Newtonian fluid with a viscosity $\mu_{1}$. For section 2 in the range of shear rates of $10^{2} \mathrm{~s}^{-1}$ to $10^{6} \mathrm{~s}^{-1}$ is characteristic decrease in viscosity as degree equations. In section 3 at a shear rate greater than $10^{6} \mathrm{~s}^{-1}$ oil behaves as a Newtonian fluid with a viscosity $\mu_{2}$.

Highly viscous boundary layer is calculated as follows [9]:

$$
\mu_{i}=\mu^{*}(T, p, \dot{\gamma})+\mu_{S} \exp \left(-h_{i} / l_{h}\right),
$$

where $l_{h}$ - characteristic parameter with dimension of length, the value of which is specific to each combination of lubricating oil and solid surface $\mu_{S}$ - parameter representing the equivalent viscosity at infinitely small distance from the boundary surface.

Thermal processes in oil film of heavy-loaded bearing are considered using the solutions of the generalized energy (heat) equation for a thin layer of viscous incompressible fluid between two moving surfaces. We used isothermal approach, which included three-constant formulas of Fogel [10]:

$$
\mu(T)=C_{1} \cdot \exp \left(C_{2} / T+C_{3}\right),
$$

where $C_{1}, C_{2}, C_{3}$ - empirical constants.

\section{Modified Reynolds equation}

The reactions of the lubricating film were determined based on the results of the numerical integration of the Reynolds equation for the pressures in the lubricating film. For a bearing with the reciprocating movement of the piston for non-Newtonian liquid, the reaction has the form:

$$
\frac{\partial}{\partial \varphi}\left[\bar{h}^{n+2} \overline{G_{1}} \bar{\rho} \frac{\partial \bar{p}}{\partial \varphi}\right]+\frac{1}{a^{2}} \frac{\partial}{\partial \bar{z}}\left[\bar{h}^{n+2} \overline{G_{1}} \bar{\rho} \frac{\partial \bar{p}}{\partial \bar{z}}\right]=\frac{\partial \bar{h}}{\partial \bar{z}}\left[\bar{\rho} \bar{h} \bar{G}_{2}\right]+\frac{\partial}{\partial \tau}(\bar{\rho} \bar{h})
$$

where $\quad \bar{G}_{1}=\left(\bar{\phi}_{2}-\bar{\phi}_{1}^{2} / \bar{\phi}_{0}\right), \quad \bar{G}_{2}=\left(1-\bar{\phi}_{1} / \bar{\phi}_{0}\right) \cdot \bar{w}, \quad \bar{\phi}_{k}=\int_{0}^{1} \bar{y}^{k} / \bar{\mu}^{*} d \bar{y}, k=0,1,2$; $\bar{p}=p \psi^{2} /\left(\mu_{0} \omega_{0}\right)$ is the dimensionless pressure in the lubricating film; $\psi=h_{0} / R$ is the relative characteristic thickness of the lubricating film; $\bar{h}=h / h_{0} ; \quad \bar{\mu}^{*}=\mu^{*} / \mu_{0} ; \quad-a \leq \bar{z} \leq a ; \quad \bar{z}=z / R ; \quad \varphi=x R ; \quad a=B /(2 R) ;$ $\bar{w}=w /(\omega R) ; \tau, \bar{h}$, and $\bar{\mu}^{*}$ are the dimensionless time, thickness of the lubricating film, and effective viscosity of the lubricant that corresponds to 
the temperature $T ; B, R$ are the width and radius of the bearing; $\mu_{0}, h_{0}$, and $\omega$ are the viscosity of the lubricant, the characteristic thickness of the lubricating film at the central position of the journal, and the rotation frequency of the journal, respectively; $w$ is the velocities of the reciprocating movement of the piston; $\bar{w}$ is the dimensionless velocity of the reciprocating movement of the piston.

When integrating eqn (4) in the domain $\Omega=(\varphi \in 0,2 \pi ; \bar{z} \in-a, a)$ the SwiftStieber boundary conditions were used; they are written in the form of the following limitations on the function $\bar{p}(\varphi, \bar{z})$ :

$$
\bar{p}(\varphi, \bar{z}= \pm a)=0 ; \bar{p}(\varphi, \bar{z})=\bar{p}(\varphi+2 \pi, \bar{z}) ; \bar{p}(\varphi, \bar{z}) \geq 0 .
$$

When calculating the trajectory of the piston on the lubricating film in the clearance of the cylinder of the ICE (Fig. 1), the coordinate system was fixed to the stationary cylinder; the origin of the moving coordinate system was placed in the center of moving piston mass.

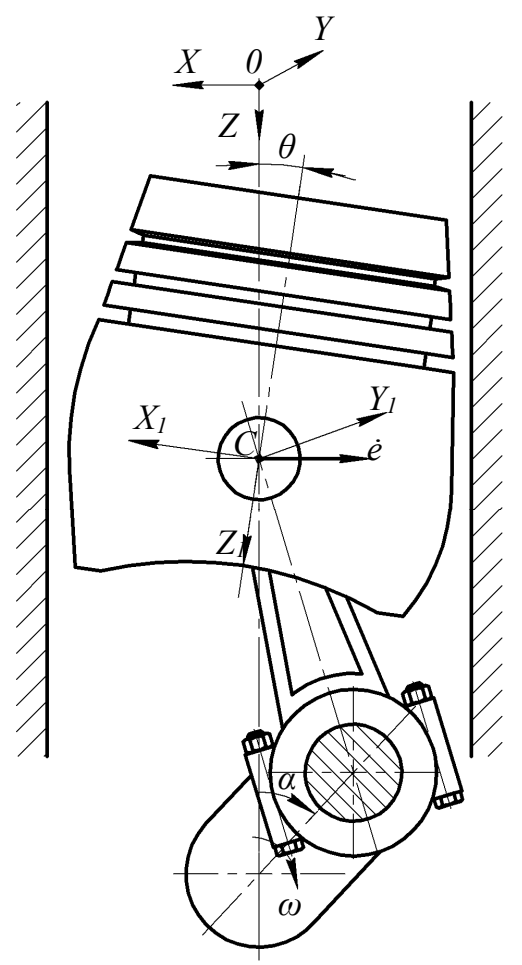

Figure 1: Dynamics of piston on lubricating film in cylinder of diesel engine. 
The thickness of the lubricating layer, taking into account the arbitrary geometry friction surfaces of piston, cylinder liner, and incline of their axes is given by

$$
\bar{h}(\varphi, \bar{z}, \tau)=\bar{h}^{*}(\varphi, \bar{z})-\chi \cos \varphi+\bar{z} \cdot \sigma / a \cdot \cos \varphi,
$$

where $\bar{h}^{*}(\varphi, \bar{z})-$ is the dimensionless film thickness at the center position of the piston in the cylinder liner with non-ideal geometry; $\chi(\tau)-$ is the dimensionless piston's eccentricity; $\sigma-$ is the dimensionless relative angle of incline of the piston.

One of the most effective methods of integrating of the Reynolds equation, using the finite difference method, is a multigrid algorithm. It allows reducing the time to solve eqn (4), which after approximation is reduced to the system of algebraic equations that are tailored to the appropriate boundary conditions and are solved. Sidel iterative method can be used as an example.

\section{The equations of movement}

Complex movements of the piston consist of its translation displacements along and across the axis of the cylinder at the velocities $\mathrm{w}$ and $\dot{e}$, respectively, as well as of the rotational movement about the axis of the piston pin at the velocity $\dot{\theta}$ (here, $e$ is the eccentricity and $\theta$ is the angle of incline of the piston). Forces of gravity and friction forces were ignored because of their small values. Thus, due to the specific features of kinematics and clearances that exist in the crank mechanism, in the general case, the piston can move in the $\mathrm{XOZ}$ and $\mathrm{YOZ}$ planes. The acceleration of the point $\mathrm{C}$ along the $\mathrm{OZ}$ axis was assumed to be equal to the acceleration of the translation movement of the piston, which was determined in the kinematics of the plane parallel movement of the crank mechanism. The force of action of the connecting rod was assumed to be a specified force that lay in the plane parallel to the $\mathrm{XOZ}$ plane; the cylinder was considered to be stationary.

Taking into account the assumptions in [11], we neglected the displacement of the piston in the YOZ plane during the analysis of the displacements of the piston in the cylinder and restricted ourselves to solving for the plane movement model. In this case, it is supposed that the piston performs plane parallel movement in the XOZ space and the equations of movement have the following form:

$$
\left\{\begin{array}{c}
m \ddot{X}_{c}=F_{x}+R_{x} ; \\
J_{y_{1}} \ddot{\theta}_{y}=M_{y_{1}}^{F}+M_{y_{1}}^{R},
\end{array}\right.
$$

where $m$ is the mass of the piston $F_{x}$ is the projection of the resultant vector of the external forces on the OX axis, $M_{y_{1}}^{F}$ is the projection of the principal moment of the external forces with regard to the center $\mathrm{C}$ on the $\mathrm{CY}_{1}$ axis, $J_{y_{1}}$ is the 
moment of inertia of the piston with regard to the $\mathrm{CY}_{1}$ axis, and $R_{x} M_{y_{1}}^{R}$ are the reaction and the moment of the reaction of the lubricating film.

System (7) was solved using the method based on backward differentiation formulas (the BDF method) described in detail in [12]. When determining the second derivatives, system (7) was reduced to the implicit difference scheme, which was solved by the Newton method. This yielded the coordinates of the center of mass of the piston along the angle of rotation of the crankshaft, i.e., the trajectory of the piston in the cylinder.

\section{Results and discussion}

To determine the rheological parameters of the eqn (1) and (8) experiments were conducted for different classes of lubricants. For the experiment, six multigrade oils of engine were chosen. The rotational viscometer with a computer controlled was used for research.

In the experiment, it was found that parameter $n$, that characterizes the degree of non-Newtonian behavior of lubricants, depends on temperature. The results are shown in Fig. 2.

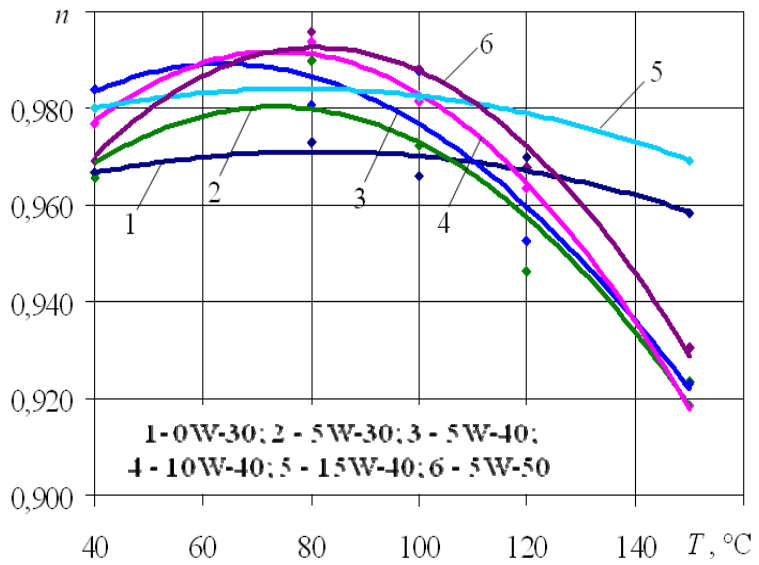

Figure 2: $\quad$ Parameter $n$ in function of the temperature.

It is common practice to consider the following main hydromechanical characteristics (HMCs) of the piston-cylinder tribosystem: the instantaneous values of the minimum thickness of the lubricating film $\inf h_{\min }$, and the maximum hydrodynamic pressure $p_{\max }(\tau)$, as well as their values $h_{\min }^{*}$ and $p_{\max }^{*}$ averaged over the cycle $\tau_{c}$; the instantaneous and average friction power loss $N(\tau)$ and $N^{*}$; the average flow rate of the lubricant towards the combustion chamber $Q^{*}$ and the cycle-averaged temperature of the lubricating film $T$. 
The calculation of the HMCs of the pair was carried out for a ChN 13/15 diesel engine with the initial data presented in Table 1. Additional initial data included the indicator diagram.

Table 2 and fig. 3(a) and (b) present the results of calculation of HMCs, which show the influence of Newtonian and non-Newtonian fluids (the influence of viscosity versus shear rate).

Table 1: Initial data.

\begin{tabular}{|l|c|c|}
\hline \multicolumn{1}{|c|}{ Parameter } & $\begin{array}{c}\text { Unit of } \\
\text { measure }\end{array}$ & Value \\
\hline Radius of crank & $\mathrm{m}$ & 0.075 \\
\hline Length of connecting rod & $\mathrm{m}$ & 0.260 \\
\hline Diameter of cylinder & $\mathrm{m}$ & 0.130 \\
\hline Diameter of piston & $\mathrm{m}$ & 0.130 \\
\hline Area of piston & $\mathrm{m}^{2}$ & 0.013 \\
\hline Mass of piston set & $\mathrm{kg}$ & 5.035 \\
\hline $\begin{array}{l}\text { Mass of parts of crank mechanism that are in } \\
\text { reciprocating movement }\end{array}$ & $\mathrm{kg}$ & 1.929 \\
\hline $\begin{array}{l}\text { Mass of parts of crank mechanism that are in rotational } \\
\text { movement }\end{array}$ & $\mathrm{kg}$ & 3.858 \\
\hline Height of piston skirt & $\mathrm{m}$ & 0.084 \\
\hline Nominal diametral clearance in piston-cylinder pair & $\mathrm{m}$ & 0.0002 \\
\hline Temperature of lubricating film & ${ }^{\circ} \mathrm{C}$ & 110 \\
\hline
\end{tabular}

Table 2: Hydromechanical characteristics of the piston-cylinder tribosystem of ChN 13/15 diesel.

\begin{tabular}{|c|c|c|c|}
\hline Parameter & Unit of measure & $\begin{array}{c}\text { Newtonian } \\
\text { lubricant }\end{array}$ & $\begin{array}{c}\text { Non-Newtonian } \\
\text { lubricant }\end{array}$ \\
\hline$N^{*}$ & $\mathrm{~W}$ & 624.3 & 513.3 \\
\hline$T$ & ${ }^{\circ} \mathrm{C}$ & 105.9 & 102.6 \\
\hline$Q^{*}$ & $1 / \mathrm{s}$ & 0.0411 & 0.0347 \\
\hline$h_{\min }^{*}$ & $\mu \mathrm{m}$ & 17.83 & 16.85 \\
\hline$p_{\max }$ & $\mathrm{MPa}$ & 11.68 & 11.96 \\
\hline$h_{\min }$ & $\mu \mathrm{m}$ & 8.6 & 7.5 \\
\hline
\end{tabular}

The results indicate that the use of structure-viscous oils leads to the reduction of power losses due to friction approximately $18 \%$. The flow rate of the lubricant towards the combustion chamber decreases by $15 \%$ and the average integral temperature decreases by $2-3^{\circ} \mathrm{C}$. However, the minimum film thickness and maximum hydrodynamic pressure decrease by an average of $5-12 \%$ and $2-4 \%$ respectively. In the hydrodynamic regime of friction the presence of adsorbed layers leads to an increase in the minimum lubricating film thickness by 40 $45 \%$, temperature of $6-7 \%$, the maximum hydrodynamic pressure by $4-5 \%$. 


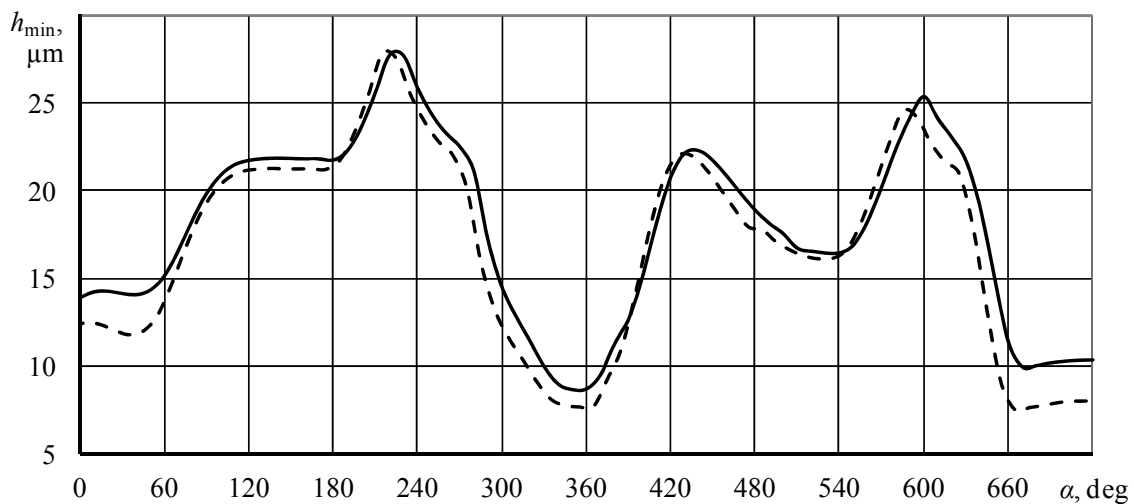

(a)

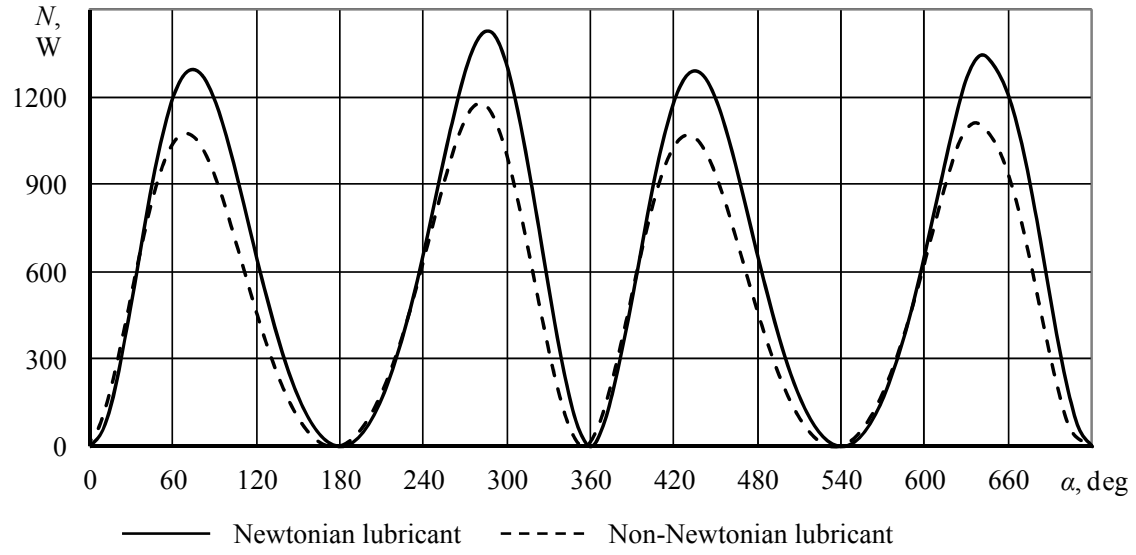

(b)

Figure 3: HMCs of the piston-cylinder tribosystem: the minimal lubricant film thickness in function of the crankangle (a); the friction power loss in function of the crankangle (b).

\section{Conclusions}

Considering one of the properties of the lubricant does not reflect the processes taking place in a thin lubricating film. Each of these properties of a lubricant and viscosity depend on one of the parameters ( $p, T, \dot{\theta}$ and more) improve or worsen the hydromechanical characteristics of tribosystem.

Therefore, the choice of the rheological models used in the calculation of heavy-loaded friction units of machines, depend on the type, operating conditions and the lubricant of a tribosystem as well as the tasks set by a design engineer. 


\section{Acknowledgements}

This work was financially supported by a grant of the Ministry of Education and Science of Russian Federation for the implementation of applied research on the lot 2014-14-579-0109. Unique identifier for Applied Scientific Research (Project) RFMEFI57714X0102. This work has been carried out within the National Research South Ural State University - grantees, the agreement № 14.577.21.0102.

The authors also express special thanks to Ksenya Volchenkova (Associate Professor of South Ural State University) for editing the translation of the paper.

\section{References}

[1] Paranjpe R.S., Analysis of Non-Newtonian Effects in Dynamically Loaded Finit Journal Bearings Including Mass Conserving Cavitation, ASME Journal of Tribology, vol. 114, pp. 736-744, 1992.

[2] Gesim B.A., Non-Newtonian Effects of Multigrade Oils on Journal Bearings Performance, Tribology Transactions, vol. 33, pp. 384-394, 1990.

[3] Chao Zhang., TEHD Behavior of Non-Newtonian Dynamically Loaded Journal Bearings in Mixed Lubrication for Direct Problem, Journal of Tribology Transactions of The ASME - J Tribol-trans, vol. 124, no. 1, (8 pages), 2002.

[4] Prokop'ev, V.N., Dynamics of complex-loaded bearing lubricated by a non-Newtonian fluid. V. Prokop'ev, A. Boyarshinova, E. Zadorozhnaya, Problems of Mechanical Engineering and reliability of the machines. № 6. pp. 108-114, 2005.

[5] Xiao-Li, Wang, Numeric analysis of journal bearings lubricated with micropolar fluids including thermal and cavitating effects. Wang Xiao-Li, Zhu Ke-Oin, J. Tribology International, V. 39, pp. 227-237, 2006.

[6] Gavrilov, K., Numerical and experimental investigations of tribosystems of piston engines taking into account boundary lubrication regime, macro and microgeometry of contact interface. K. Gavrilov, Y. Rozhdestvensky, Society of tribologists and lubrication engineers, Annual Meeting \& Exhibition, 2, pp. 16-24, 2014.

[7] Prokop'ev, V.N., Hydromechanical characteristics of con-rod bearings lubricating Non-Newtonian liquids. V. Prokop'ev, A. Boyarshinova, E. Zadorozhnaya, K. Gavrilov, I. Andryuschenko, Bulletin of SUSU, a series of "Mechanical Engineering”, № 1 (41), pp. 17-24, 2005.

[8] Zadorozhnaya, E., Rheological Model of a Boundary Layer of Lubricant, E. Zadorozhnaya, I. Levanov, I. Mukhortov. STLE $66^{\text {th }}$ STLE Annual Meeting \& Exhibition, 15-19 May, Atlanta, Georgia (USA), 2011.

[9] Mukhortov, I.V. Multilayer adsorption of lubricants and applied to the theory of fluid friction, Bulletin of SUSU, a series of "Mechanical Engineering”, edition 18, № 31 (248), pp. 62-67, 2011. 
508 High Performance and Optimum Design of Structures and Materials II

[10] Prokop'ev, V., Dynamics and Lubrication of Tribounits of Piston and Rotary Machines: Monograph. V. Prokop'ev, Y. Rozhdestvensky, E. Zadorozhnaya et al. South Ural State University, ISBN 978-5-696-040363, Chelyabinsk vol. 2., 212 pp., 2011.

[11] Goryacheva I.G., Morozov A.V., Rozhdestvensky Y.V., Gavrilov K.V., Doikin A.A., Development of method for calculating and experimentally evaluating tribological parameters of piston-cylinder tribosystem of diesel engine. Journal of Friction and Wear, V.34, № 5, pp. 339-348, 2013.

[12] Prokop'ev, V.N., Rozhdestvenskii, Yu.V., and Shirobokov, N.V., Increasing of effectiveness of algorithms of outlet parameters of in a complicated manner loaded sliding supports of transport machines engines, Vestnik Ural. Mezhregion. Otd. Ross. Akad. Transporta, № 2, p. 28, 1999. 